\title{
Computational insights into electrochemical cross-coupling of quaternary borate salts
}

\author{
Florian Matz, ${ }^{*, \dagger, \ddagger}$ Arif Music, ${ }^{\llbracket}$ Dorian Didier, $₫$ and Thomas-C. Jagau ${ }^{*, \dagger}$ \\ $\dagger$ Division of Quantum Chemistry and Physical Chemistry, KU Leuven, \\ Celestijnenlaan 200F, 3001 Leuven, Belgium \\ $\ddagger$ Institute for Physical and Electrochemistry, Leibniz University Hanover, Callinstrasse 3A, \\ 30167 Hanover, Germany \\ 9Department of Chemistry, Ludwig Maximilian University of Munich, \\ Butenandtstrasse 5-13, 81377 Munich, Germany \\ E-mail: florian.matz@pci.uni-hannover.de; thomas.jagau@kuleuven.be
}

\begin{abstract}
Cross-coupling reactions for $\mathrm{C}-\mathrm{C}$ bond formation represent a cornerstone of organic synthesis. In most cases, they make use of transition metals, which has several downsides. Recently, metal-free alternatives relying on electrochemistry have gained interest. One example of such a reaction is the oxidation of tetraorganoborate salts that initiates aryl-aryl and aryl-alkenyl couplings with promising selectivities. This work investigates the mechanism of this reaction computationally using density functional and coupledcluster theory. Our calculations reveal a distinct difference between aryl-alkenyl and aryl-aryl couplings: While C-C bond formation occurs irreversibly and without an energy barrier if an alkenyl residue is involved, many intermediates can be identified in aryl-aryl couplings. In the latter case, intramolecular transitions between reaction paths leading to different products are possible. Based on the energy differences be-
\end{abstract}


tween these intermediates, we develop a kinetic model to estimate product distributions for aryl-aryl couplings.

\section{Introduction}

Many years after the development of transition metal catalyzed cross-coupling reactions, they are now broadly used in organic synthesis. While palladium catalysts have been most popular since the introduction of these reactions, ${ }^{1,2}$ a large variety of noble metals are in use today. In addition, catalysis by non-noble transition metals has gained interest too. ${ }^{3}$ In such reactions, residues can be introduced to the metal scaffold by insertion to an aryl-halogen bond and by ligand exchange with a precursor. The coupling then happens via a reductive elimination, which is facilitated by transition metals due to their unoccupied d orbitals. Incorporating transition metals in organic reactions is, however, accompanied by several disadvantages: first, they are expensive and large amounts of catalyst are lost in the workup process. Second, the lost catalyst is environmentally problematic and can pose toxicity issues to the consumers if remaining in the products. Reductive eliminations occur, however, also in metal-free compounds, as for example in the oxidized tetraphenylborate anion, which can produce biphenyl. ${ }^{4,5}$ These reactions form the basis for an attractive synthetic route to C-C coupling products that avoids the use of transition metal catalysts.

In our previous work with a focus on the experimental side, we investigated substituted tetraphenylborates ${ }^{6}$ and alkenyltriarylborates. ${ }^{7}$ The oxidation of the borate salts was perfomed electrochemically with RVC electrodes in acetonitrile and in a subsequent work by means of a photocatalyst. ${ }^{8}$ Similar reactions occur with chemical oxidants. ${ }^{9}$ For aryl-aryl couplings, we proposed ${ }^{6}$ the mechanisms shown in Fig. 1 based on cyclic voltammetry, galvanostatic measurements, and density functional theory (DFT) calculations.

The initial oxidation of the quaternary borate anion results in a doublet state named

A as shown for tri(fluorophenyl)methoxyphenylborate in Fig. 1. For clarity, charges and 


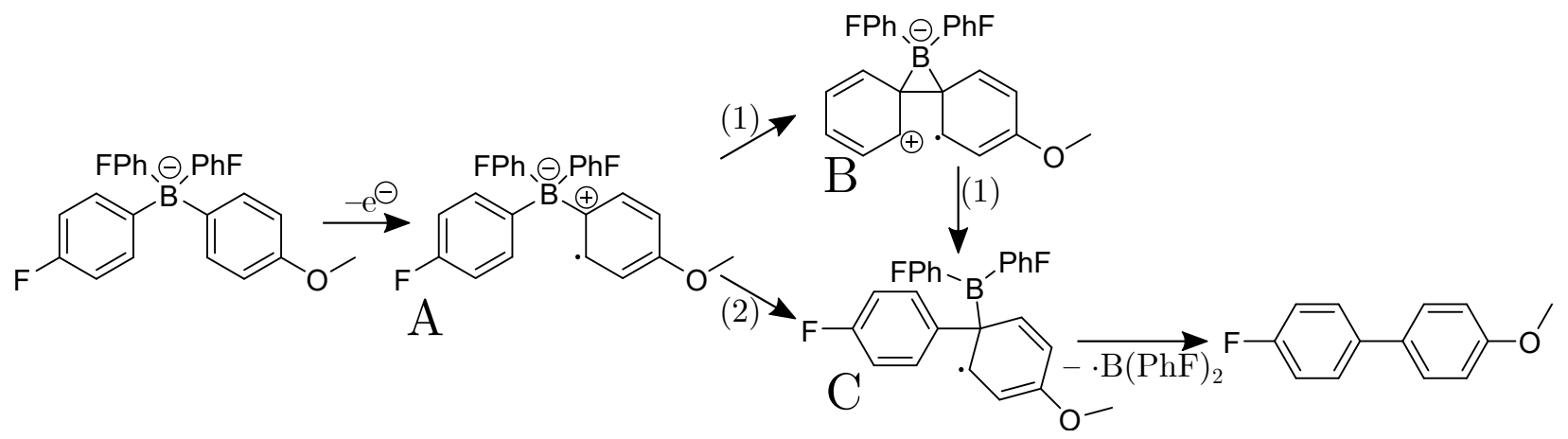

Figure 1: Proposed mechanism for aryl-aryl coupling in tri(fluorophenyl)methoxyphenylborate.

radical electrons are depicted as localized in this figure and in the rest of this manuscript, although they are in reality delocalized over the conjugated $\pi$ systems. In structure $\mathbf{A}$, there is no bond between any two of the four substituents at the boron atom. DFT calculations revealed that at this point, the spin density is centered on the most electron-rich moiety. Following pathway (1), the three-membered ring $\mathbf{B}$, which features a bond between two of the substituents, is formed by $\pi$-stacking and subsequently opens to structure $\mathbf{C}$, where the boron residue is bound to only one side of the coupling product. The alternative mechanism (2) proceeds directly from $\mathbf{A}$ to $\mathbf{C}$ via $\sigma$-bond cleavage and was also proposed for aryl-vinyl coupling reactions. ${ }^{6-8}$ Subsequently, the boron-carbon bond in $\mathbf{C}$ is broken homolytically, furnishing the final product and removing the radical character. As a side product, borinic acids $\mathrm{BR}_{2} \mathrm{OH}$ are formed.

In this work, we explore the reaction mechanism in detail. By means of DFT and coupledcluster (CC) calculations, we characterize the reaction path and identify intermediates with a special focus on differences between aryl-aryl and aryl-alkenyl couplings. Based on the energetics of the intermediates $\mathbf{B}$, we predict product distributions for heterosubstituted molecules and compare them with experimental data. In addition, we model reaction paths for the elimination of the coupling product from intermediate $\mathbf{C}$ and investigate the role of the solvent in this reaction step. 


\section{Computational details}

To obtain a qualitative picture of the reaction path, we carried out calculations with the Gaussian ${ }^{10}$ and Q-Chem ${ }^{11}$ program packages at the B3LYP/6-31G(d) level of theory. ${ }^{12}$ In this way, we identified stable structures along the reaction path for a large set of borate salts comprising $\mathrm{BVi}_{4}, \mathrm{BVi}_{3} \mathrm{St}, \mathrm{BVi}_{2} \mathrm{St}_{2}, \mathrm{BVi}_{3} \mathrm{Ph}, \mathrm{BPh}_{2} \mathrm{Vi}_{2}, \mathrm{BPh}_{3} \mathrm{Vi}, \mathrm{BPh}_{2} \mathrm{St}_{2}, \mathrm{BPh}_{3} \mathrm{St}, \mathrm{B}(\mathrm{PhF})_{3} \mathrm{St}$,

$\mathrm{BPh}_{4}, \mathrm{~B}(\mathrm{PhF})_{4}, \mathrm{~B}(\mathrm{PhOMe})_{2}(\mathrm{PhF})_{2}, \mathrm{~B}(\mathrm{PhF})_{3}(\mathrm{PhOMe})$, and $\mathrm{B}(\mathrm{PhF})_{3}($ ortho-PhOMe) where $\mathrm{Vi}=$ vinyl, $\mathrm{St}=$ styryl, $\mathrm{Ph}=$ phenyl and all $\mathrm{F}$ and OMe substituents are in para-position if not indicated otherwise. This set is chosen to explain the most prominent trends observed in experiment. Our previous work ${ }^{6,7}$ showed clearly different product distributions when comparing alkenyl with aryl groups, electron-rich with electron-poor aryl residues, and aryl rings with different patterns. We thus investigate here borate salts with vinyl groups of different sizes, borate salts with a different number of the electron-rich PhOMe and the electron-poor PhF group, and borate salts that differ only in the position in which the aryl residues are substituted.

For each molecule, we optimized the anionic equilibrium structures first. Notably, multiple minima were found in some cases. Starting at these structures we followed the gradient on the potential energy surfaces (PES) of the oxidized neutral molecules. To locate minima and first-order saddle points, we used PES scans and the freezing string method. ${ }^{13}$ Stationary points were characterized by frequency calculations that also yielded vibrational contributions to the enthalpy and entropy from which Gibbs free energies and, subsequently, barrier heights were computed. In the following, all barrier heights are given as Gibbs free activation energies.

After these initial calculations, we reexamined selected molecules and reaction steps with the hybrid-GGA functional $\omega \mathrm{B} 97 \mathrm{X}-\mathrm{D},{ }^{14}$ the one-parameter hybrid-meta GGA functional TPSSh, ${ }^{15}$ and the empirical hybrid-meta GGA functional M06-2X. ${ }^{16}$ All DFT calculations were carried out with the SG-2 grid. ${ }^{17}$ In addition, we investigated the impact of the conductor-like polarizable continuum model (C-PCM) for solvation ${ }^{18}$ and D3 dispersion 
corrections. ${ }^{19,20}$ Furthermore, we performed selected single-point calculations with equationof-motion ionization potential coupled-cluster singles and doubles ${ }^{21,22}$ (EOM-IP-CCSD) and the 6-311G $(\mathrm{d}, \mathrm{p})$ basis set at geometries obtained with B3LYP/6-31G(d). In these calculations, we chose the anion as CC reference state.

\section{Results and discussion}

Our investigations identified two different reaction paths that are exemplified in Fig. 2. Whenever alkenyl groups are part of the coupling product, the reaction is kinetically unhindered, regardless of whether an alkenyl or an aryl group is transferred. This can be seen from Fig. 1 in the supporting information where the coupling of two styryl residues and that of one styryl and one phenyl residue are compared. Meanwhile, substantial barriers are encountered in aryl-aryl couplings. These computational results confirm our earlier hypothesis presented in Fig. 1. A new aspect is the formation of a further stable structure $\mathbf{D}$ from $\mathbf{C}$ in which the boron residue has moved to an adjacent carbon atom through a secondary 1,2 -rearrangement.

\section{Couplings involving alkenyl groups}

Borates substituted with St or Vi groups exhibit reaction paths without any significant barriers after oxidation; this is exemplified for $\mathrm{BPh}_{2} \mathrm{St}_{2}$ in the right panel of Fig. 2. Especially substrates with bare Vi groups show no barrier at all for rearrangement from $\mathbf{A}$ to $\mathbf{D}$ whereas tiny barriers are sometimes observed when other alkenyl groups are involved. The threemembered ring $\mathbf{B}$ is formed but does not represent a stable structure; it is only associated with an energy plateau. The relaxation from $\mathbf{A}$ to $\mathbf{C}$ is distinctly exergonic. As an example, $135 \mathrm{~kJ} \mathrm{~mol}^{-1}$ and $154 \mathrm{~kJ} \mathrm{~mol}^{-1}$, respectively, are released in St-St and St-Ph coupling starting from $\mathrm{BPh}_{2} \mathrm{St}_{2}{ }^{-}$. Energy differences for other molecules are very similar and can be found in Table 1 of the supporting information. A further stable structure $\mathbf{D}$, which is an isomer of $\mathbf{C}$ 


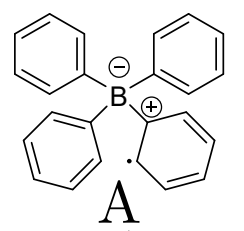

$0 \mathrm{~kJ} / \mathrm{mol}$

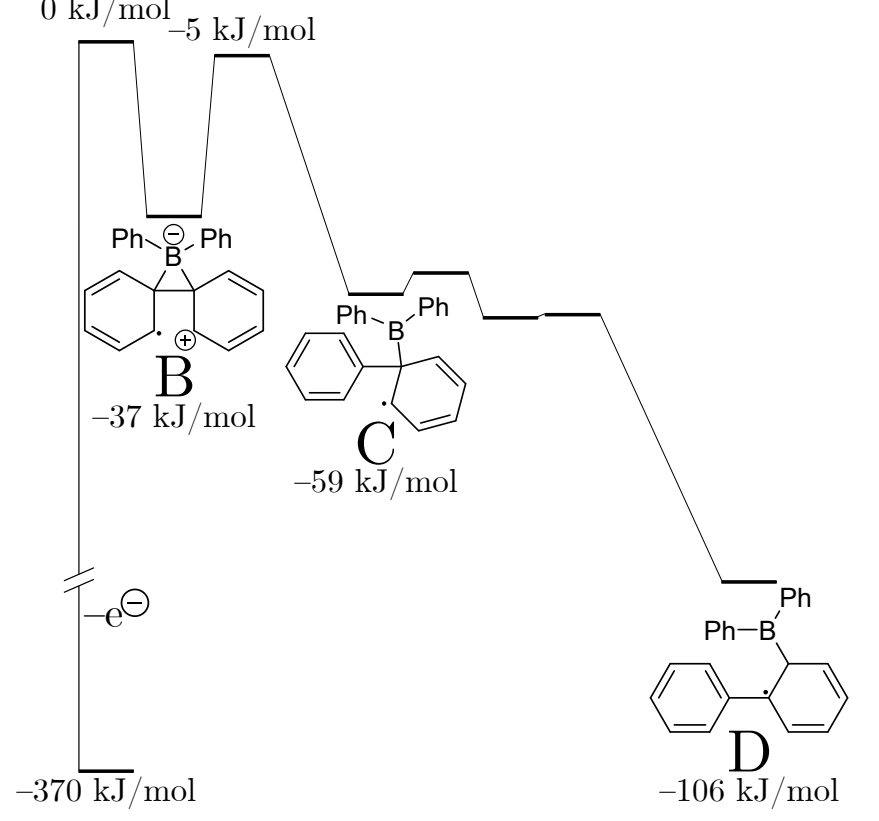

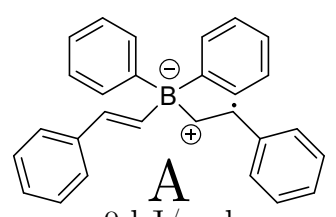

$0 \mathrm{~kJ} / \mathrm{mol}$

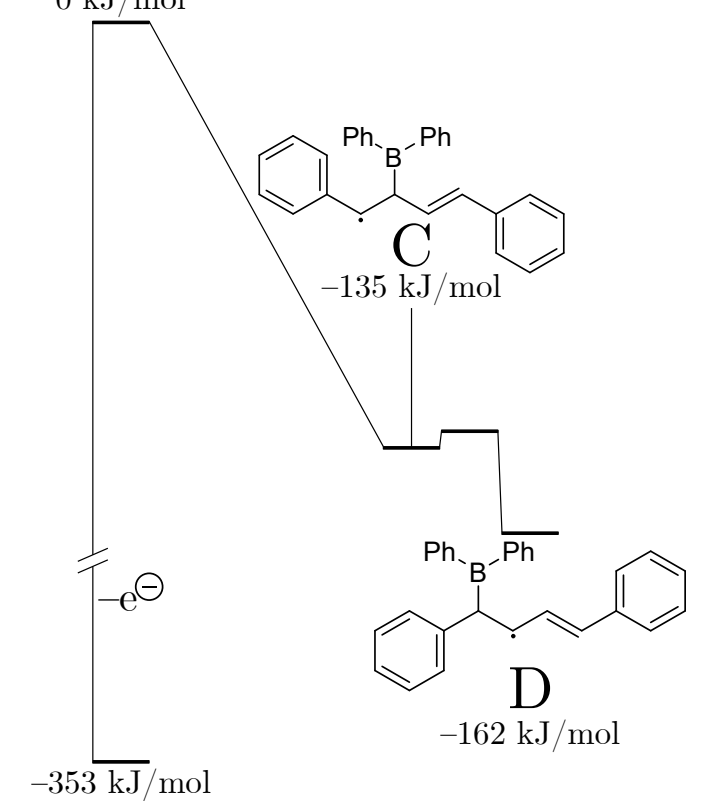

Figure 2: Left: Formation of biphenyl from tetraphenylborate. The three-membered ring $\mathbf{B}$ is formed as an intermediate. Right: Formation of 1,4-diphenylbutadiene from diphenyldistyrylborate. No significant barrier was found. Numbers represent Gibbs free energies $G$.

with the boron atom bound to the terminal end of the newly formed diene, was identified in St-St coupling. $\mathbf{D}$ is somewhat lower in energy than $\mathbf{C}\left(\Delta G=-28 \mathrm{~kJ} \mathrm{~mol}^{-1}\right)$ and the barrier between the two structures is only $8 \mathrm{~kJ} \mathrm{~mol}^{-1}$. In contrast, migration of the boron residue along the phenyl ring is not favorable in the case of St-Ph coupling, presumably because the aromaticity of the phenyl group would be lost.

To obtain different coupling products from the same substrate in these calculations, we modified the anionic starting geometries. However, we were unable to obtain all possible products in this way: Neither $\mathrm{Ph}-\mathrm{Ph}$ nor Vi-Vi coupling takes place if a St group is present, which we attribute to the more extended delocalization of the unpaired electron in the St group. From these results we expect that the coupling proceeds without delay after the initial oxidation and that the formation rate of the product is determined by the oxidation rate. 
This implies that the product distribution is hard to predict. Electronic energy differences between the anionic precursor structures likely play a role but the vibrational state of the anion at the moment of oxidation may also be relevant because this could make an impact on the direction in which the oxidized molecule moves on the neutral PES.

\section{Aryl-aryl couplings}

For aryl-aryl couplings, the three-membered ring B is kinetically stabilized for all molecules that we examined, as exemplified for tetraphenylborate in the left panel of Fig. 2. This reflects the steric crowding: the three-membered ring is substituted with 6 carbon atoms that have to move past each other when the ring opens. The enthalpies associated with the initial relaxation from $\mathbf{A}$ to $\mathbf{B}$ fall in a range from -63 to $-27 \mathrm{~kJ} \mathrm{~mol}^{-1}$ and are thus distinctly smaller than when an alkenyl group is involved. Generally, more energy is gained if more electron-rich substituents are coupled. Enthalpies computed for five different tetraarylborates are available from Tables $2-4$ in the supporting information. Similar to aryl-alkenyl couplings, we found that the coupling of two electron-deficient rings, for example of two $\mathrm{PhF}$ moieties in $\mathrm{B}(\mathrm{PhOMe})_{2}(\mathrm{PhF})_{2}^{-}$is not possible; the intermediate $\mathbf{B}$ is not stable in such cases.

The barrier towards ring opening of $\mathbf{B}$ as calculated by B3LYP is in the range of 28 to $50 \mathrm{~kJ} \mathrm{~mol}^{-1}$ with the higher values in general corresponding to electron-rich substituents, for example, $49 \mathrm{~kJ} \mathrm{~mol}^{-1}$ for formation of $\mathrm{MeOPh}-\mathrm{PhOMe}$ from $\mathrm{B}(\mathrm{PhOMe})_{2}(\mathrm{PhF})_{2}^{-}$and $35 \mathrm{~kJ} \mathrm{~mol}^{-1}$ for formation of $\mathrm{FPh}-\mathrm{PhF}$ from $\mathrm{B}(\mathrm{PhF})_{4}^{-}$. Because of the importance of this reaction step for the selectivity of the coupling (see next Section), we computed the electronic energies for the ring opening of $\mathrm{BPh}_{4}$ also with EOM-IP-CCSD/6-311G(d,p) using the anion as CC reference state. Together with vibrational contributions from B3LYP, this resulted in a value of $20 \mathrm{~kJ} \mathrm{~mol}^{-1}$ for the barrier height as compared to $28 \mathrm{~kJ} \mathrm{~mol}^{-1}$ obtained with B3LYP for this molecule. If one ring is substituted in ortho-position, the relaxation from $\mathbf{A}$ to $\mathbf{B}$ releases less energy compared to the corresponding para-substituted compound, while more energy is gained from $\mathbf{B}$ to $\mathbf{C}$ and the barrier for opening the cyclic intermediate 
$\mathbf{B}$ is lower. We attribute this destabilization of $\mathbf{B}$ to repulsive interaction between the substituent in ortho-position and the second aryl ring. Similar to aryl-alkenyl couplings, a further intermediate $\mathbf{D}$, in which the boron residue is bound to the ortho-position, also exists in aryl-aryl couplings (see Fig. 2). The relaxation from $\mathbf{C}$ to $\mathbf{D}$ is accompanied by a substantial energy gain of ca. $50 \mathrm{~kJ} \mathrm{~mol}^{-1}$ whereas further rearrangements resulting from migration to the meta- and para-position bring only negligible energy gains. If a methoxy substituent is bound to the aryl ring in ortho-position, additional cyclic intermediates similar to $\mathbf{C}$ and $\mathbf{D}$ may exist; these are depicted in Fig. 3. While all tested DFT methods found the intermediate $\mathbf{D}^{\prime}$ and predicted a barrier of ca. $15 \mathrm{~kJ} \mathrm{~mol}^{-1}$ between $\mathbf{D}$ and $\mathbf{D}^{\prime}$, only dispersion corrected methods identified C' as a stable structure.

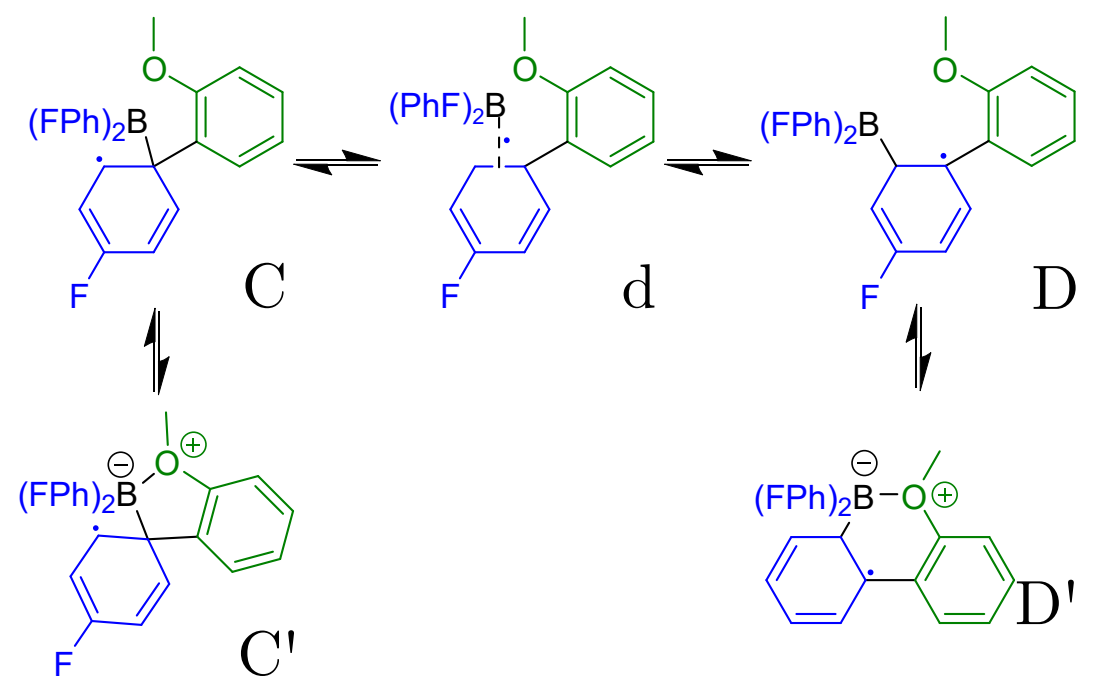

Figure 3: Stable intermediates of types $\mathbf{C}$ and $\mathbf{D}$ in the formation of $\mathrm{FPh}-\mathrm{PhOMe}$ from $\mathrm{B}(\mathrm{PhF})_{3}(\text { ortho }-\mathrm{PhOMe})^{-}$.

\section{Selectivity of aryl-aryl couplings}

The activation energy necessary to open the cyclic intermediate $\mathbf{B}$ explains the selectivity of the coupling observed for heterosubstituted tetraarylborates. Since different intermediates B are separated from each other only by low barriers, the most thermodynamically favorable species forms before the reaction proceeds. In a borate that is substituted by three identical 
aryl groups and a fourth one that is different, a transition between two intermediates that correspond to homo- and heterocoupling is possible if the $\mathrm{C}-\mathrm{C}$ bond in one $\mathrm{B}$-type structure is broken and a new $\mathrm{C}-\mathrm{C}$ bond between the two previously uninvolved residues is formed at the same time. This is shown in Fig. 4 for two isomers of $\mathrm{B}(\mathrm{PhF})_{3} \mathrm{PhOMe}$. We note, however, that B3LYP possibly underestimates the activation barriers of these transitions. For $\mathrm{BPh}_{4}^{-}$, the electronic contribution to the barrier is $23 \mathrm{~kJ} \mathrm{~mol}^{-1}$ higher when computed with EOM-IP-CCSD/6-311G(d,p) compared to B3LYP/6-31G(d).

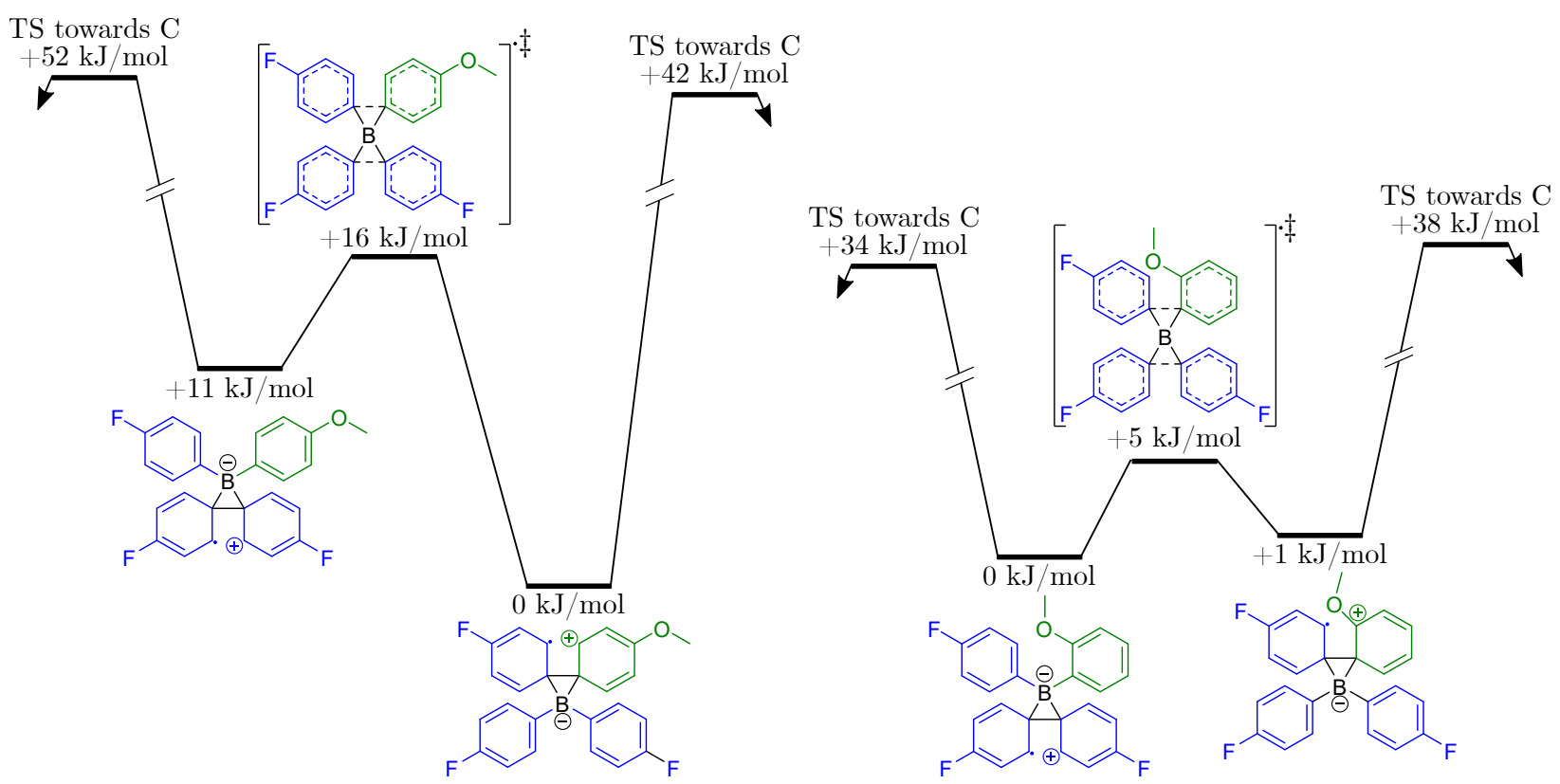

Figure 4: Transition between two $\mathbf{B}$ intermediates that correspond to formation of $\mathrm{PhF}-\mathrm{PhF}$ and $\mathrm{PhF}-\mathrm{PhOMe}$, respectively, from $\mathrm{B}(\mathrm{PhF})_{3} \mathrm{PhOMe}^{-}$. Left panel: OMe residue in paraposition. Right panel: OMe residue in ortho-position. For comparison, the energies of the transition states for the progress of the reaction from $\mathbf{B}$ to $\mathbf{C}$ are also shown.

In a borate substituted with two pairwise identical substituents, the mechanism displayed in Fig. 4 corresponds to a transition between two homo-coupled products. The transition from a $\mathbf{B}$ intermediate that leads to a homo-coupled product to one that leads to a hetero-coupled product occurs through a two-step mechanism shown in Fig. 5. The structures asym-B-hetero and asym-B-homo are available from Fig. 3 and 4 in the supporting information. Similar to the previous mechanism, EOM-IP-CCSD/6-311G(d,p) raises the activation barriers compared to B3LYP/6-31G(d), here by ca. $10 \mathrm{~kJ} \mathrm{~mol}^{-1}$. 


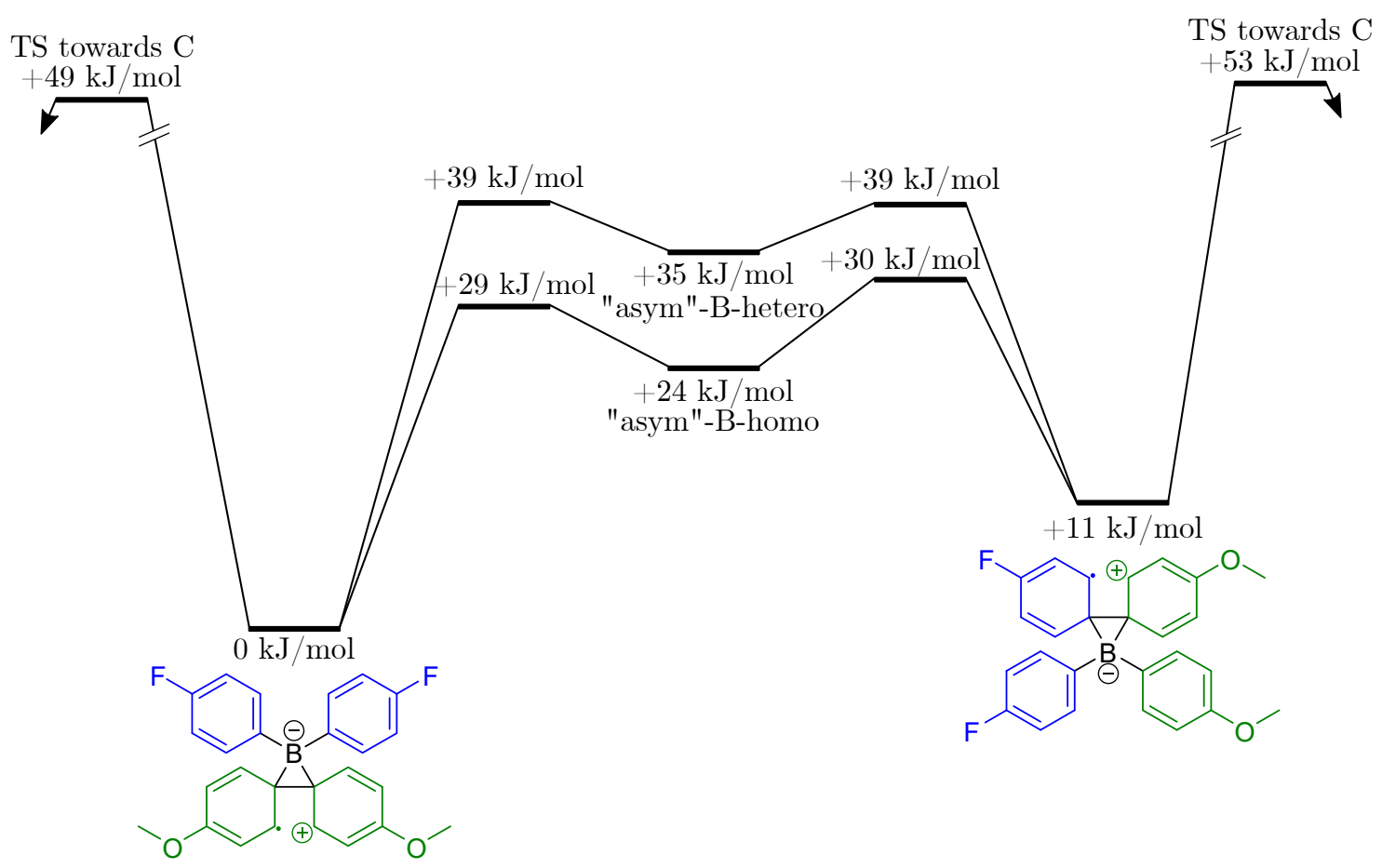

Figure 5: Transition between two $\mathbf{B}$ intermediates that correspond to formation of $\mathrm{MeOPh}-\mathrm{PhOMe}$ and $\mathrm{PhF}-\mathrm{PhOMe}$, respectively, from $\mathrm{B}(\mathrm{PhOMe})_{2}(\mathrm{PhF})_{2}^{-}$. In each case, one elementary step consists in the rotation of one of the inactive rings by 90 degrees to arrive at the asym-B arrangement of the phenyl groups with local symmetry $\mathrm{C}_{\mathrm{v}}$. The second step is a concerted rotation of all phenyl groups at once. For comparison, the energies of the transition states for the progress of the reaction from $\mathbf{B}$ to $\mathbf{C}$ are also shown.

Assuming that the equilibration between different intermediates $\mathbf{B}$ occurs faster than their decay, which results in a constant ratio between their amounts, the product distribution can be calculated as follows. We assume that each product $i$ is formed through two independent, parallel, and irreversible first-order reactions such that $\left.\mathrm{d} n_{i} / \mathrm{d} t=n_{i}^{\prime} k_{i \mathrm{a}}+n_{i}^{\prime} k_{i \mathrm{~b}}\right)$. Here, $k$ is the rate constant for ring opening, which can occur in two different directions designated by a and b. $n$ refers to the amount of product and $n^{\prime}$ to that of $\mathbf{B}$ intermediate. Under the further assumption of a steady-state process, that is, $n_{i} / n_{j}=\frac{\mathrm{d} n_{i}}{\mathrm{~d} t} / \frac{\mathrm{d} n_{j}}{\mathrm{~d} t}$ and $n_{i}^{\prime}=$ const., we obtain the following general expression for the distribution between homo-coupled and hetero-coupled products obtained from a borate that is substituted by three identical aryl 
groups and a fourth one that is different.

$$
\frac{n_{1}}{n_{2}}=\frac{n_{1}^{\prime} k_{1 \mathrm{a}}+n_{1}^{\prime} k_{1 \mathrm{~b}}}{n_{2}^{\prime} k_{2 \mathrm{a}}+n_{2}^{\prime} k_{2 \mathrm{~b}}}=\frac{n_{1}^{\prime}}{n_{2}^{\prime}} \frac{k_{1 \mathrm{a}}+k_{1 \mathrm{~b}}}{k_{2 \mathrm{a}}+k_{2 \mathrm{~b}}}
$$

Here, indices 1 and 2 refer to hetero-coupled and homo-coupled products. By means of the Eyring equation ${ }^{23}$

$$
k_{i}=\kappa_{i} N_{A} \frac{k_{\mathrm{B}} T}{h} \exp \left[-\frac{\Delta G_{i}^{\ddagger}}{R T}\right]
$$

and assuming $\kappa$ to be identical for all involved reactions, we arrive at

$$
\frac{n_{1}}{n_{2}}=\exp \left[-\frac{\Delta G^{\prime}}{R T}\right] \frac{\exp \left[-\Delta G_{1 \mathrm{a}}^{\ddagger} / R T\right]+\exp \left[-\Delta G_{1 \mathrm{~b}}^{\ddagger} / R T\right]}{\exp \left[-\Delta G_{2 \mathrm{a}}^{\ddagger} / R T\right]+\exp \left[-\Delta G_{2 \mathrm{~b}}^{\ddagger} / R T\right]}
$$

Here, we took into account the free energy differences between the $\mathbf{B}$ intermediates $\Delta G^{\prime}=$ $G_{\text {hetero }}^{\prime}-G_{\text {homo }}^{\prime}$ which determine $n_{1}^{\prime} / n_{2}^{\prime}$ and the barriers $\Delta G_{i}^{\ddagger}$ for ring opening towards $\mathbf{C}$ which yield the equilibrium constants with the activated complexes. This shows that the product distribution is influenced by a thermodynamic and a kinetic factor. For a borate substituted with two pairwise identical substituents, an additional combinatory factor of four results from the number of possible combinations of two particular residues in a heterocoupling.

Table 1: Theoretical and experimental product distributions for four heterosubstituted tetraarylborates. Theoretical results were computed from free energies obtained with B3LYP/6-31G(d). Indices 1 and 2 refer to hetero-coupled and homo-coupled product. See text for further explanation.

\begin{tabular}{lrrr|c}
\hline Molecule & \multicolumn{3}{c}{ theory } & \multicolumn{2}{c}{ experiment } \\
& $n_{1}^{\prime} / n_{2}^{\prime}$ & $\frac{k_{\mathrm{a} a}+k_{1 \mathrm{~b}}}{k_{2 \mathrm{a}}+k_{2 \mathrm{~b}}}$ & $n_{1} / n_{2}$ & $n_{1} / n_{2}$ \\
\hline $\mathrm{B}(\mathrm{PhF})_{3}(\mathrm{PhOMe})^{-}$ & 79.788 & 0.28 & 22.48 & 32.50 \\
$\mathrm{~B}(\mathrm{PhF})_{3}\left(\text { ortho }^{-} \mathrm{PhOMe}\right)^{-}$ & 0.619 & 0.21 & 0.13 & 0.42 \\
$\mathrm{~B}(\mathrm{PhOMe})_{2} \mathrm{Ph}_{2}^{-\mathrm{a}}$ & 0.022 & 30.53 & 0.66 & 1.25 \\
$\mathrm{~B}(\mathrm{PhF})_{2}(\mathrm{PhOMe})_{2}^{-\mathrm{a}}$ & 0.039 & 14.45 & 0.56 & - \\
\hline
\end{tabular}

${ }^{\mathrm{a}}$ For this molecule, homo refers to MeOPh-PhOMe.

Product distributions for four tetraarylborates obtained with B3LYP/6-31G(d) are compiled in Tab. 1 and compared with experiment. The experimental values were obtained 
with a procedure described in Ref. 6 and in the supporting information. The values for the experimental product distributions are accurate to ca. $3 \%$, i.e., the uncertainty in the experiment is much smaller than the deviation between computed and experimental values which differ by a factor between 1.4 and 3.2. However, the trend between the molecules is reproduced correctly. While the amount of homo-coupled product is overestimated by the calculations for all molecules in Tab. 1, there is no clear trend with respect to the electron richness of the coupled rings. Owing to the exponential dependence the product distribution is very sensitive to small changes in the energy differences. To test the validity of the results compiled in Tab. 1, we re-evaluated the product distributions for $\mathrm{B}(\mathrm{PhOMe})_{2}(\mathrm{PhF})_{2}^{-}$, $\mathrm{B}(\mathrm{PhF})_{3}(\mathrm{PhOMe})^{-}$, and $\mathrm{B}(\mathrm{PhF})_{3}(\text { ortho-PhOMe })^{-}$using free energies obtained using different density functionals and with EOM-IP-CCSD. The results for the product distributions can be found in table 2, underlying energies as well as values for the two factors constituting the overall product distribution are available from Tables 6-19 in the supporting information.

Table 2: Product distributions for (a) $\mathrm{B}(\mathrm{PhOMe})_{2}(\mathrm{PhF})_{2}^{-}$, (b) $\mathrm{B}(\mathrm{PhF})_{3}(\mathrm{PhOMe})^{-}$, and (c) $\mathrm{B}(\mathrm{PhF})_{3}$ (ortho-PhOMe) $)^{-}$calculated with different methods. The extrapolated experimental value for $\mathrm{B}(\mathrm{PhOMe})_{2}(\mathrm{PhF})_{2}^{-}$is obtained from the value for $\mathrm{B}(\mathrm{PhOMe})_{2} \mathrm{Ph}_{2}^{-}$scaled with a factor for the different substitution calculated with B3LYP/6-31G(d).

\begin{tabular}{|c|c|c|c|c|}
\hline Molecule & \multicolumn{2}{|c|}{ (a) } & (b) & (c) \\
\hline Distribution & $\frac{n(\mathrm{FPh}-\mathrm{PhOMe})}{n(\mathrm{MeOPh}-\mathrm{PhOMe})}$ & $\frac{n(\mathrm{MeOPh}-\mathrm{PhOMe})}{n(\mathrm{FPh}-\mathrm{PhF})}$ & $\frac{n(\mathrm{FPh}-\mathrm{PhOMe})}{n(\mathrm{FPh}-\mathrm{PhF})}$ & $\frac{n(\mathrm{FPh}-\mathrm{PhOMe})}{n(\mathrm{FPh}-\mathrm{PhF})}$ \\
\hline extrapolated experimental value & 1.05 & - & 32.5 & 0.422 \\
\hline B3LYP /6-31G(d) & 0.56 & - & 22.5 & 0.132 \\
\hline B3LYP-PCM /6-31G(d) & 9.96 & 10.7 & 33.8 & 0.327 \\
\hline B3LYP-PCM-D3/6-31G(d) & 0.41 & 22.7 & 4.4 & 0.012 \\
\hline B3LYP-PCM/6-311G(2d,p) & 0.50 & 8.6 & 13.8 & 0.010 \\
\hline B3LYP-PCM-D3/6-311G(2d,p) & 0.40 & 116.1 & 0.1 & 0.007 \\
\hline TPSSh-PCM /6-31G(d) & 16.83 & 6.3 & 138.7 & 0.316 \\
\hline TPSSh-PCM-D3/6-31G(d) & 0.58 & 4.4 & 18.2 & 0.013 \\
\hline$\omega B 97 X-D-P C M / 6-31 G(d)$ & 0.31 & 19.2 & 9.5 & 0.017 \\
\hline M06-2X-PCM/6-31G(d) & 0.19 & 19.2 & 8.6 & 0.378 \\
\hline M06-2X-PCM-D3/6-31G(d) & 0.18 & 13.7 & 10.6 & 0.359 \\
\hline EOM-IP-CCSD /6-311G(d,p) & 0.16 & - & 41.6 & 0.161 \\
\hline
\end{tabular}

Although the reaction paths obtained with the different methods do not differ qualitatively, the energetic differences between the methods are large enough to give rise to 
completely different product distributions as Tab. 2 illustrates. While a few methods produce good results for individual molecules, no approach delivers good results for all three molecules. The performance of the initially employed B3LYP/6-31G(d) method without D3 correction and PCM is relatively consistent and in qualitative agreement with CCSD/6$311 \mathrm{G}(\mathrm{d}, \mathrm{p})$. The same can be said about M06-2X/6-31G(d) with dispersion correction and PCM included. This approach predicts, however, substantially higher barriers for the transition between different $\mathbf{B}$ intermediates. It is also noteworthy that the energy differences between different $\mathbf{B}$ structures computed with different approaches are in better agreement than the activation energies. However, since there is no universal correlation between the thermodynamic and the kinetic factor, it is not possible to calculate the product distributions without accurate values for both factors.

In any case, a quantitative assessment of the product distributions is thus not possible with our current theoretical approach. We are, however, able to offer a qualitative explanation of the experimentally observed selectivity of aryl-aryl couplings that involves two factors: On the one hand, the intermediate $\mathbf{B}$ is stabilized if electron-rich rings participate in the coupling but, on the other hand, this effect is partly compensated by the relatively high barriers associated with ring opening towards an electron-rich product. As illustrated by Tab. 1 and corroborated by further results obtained using other density functionals (see Tables 16-19 in the supporting information), the first factor is more important in general. For example, for $\mathrm{B}(\mathrm{PhF})_{3}(\mathrm{PhOMe})$, the formation of $\mathrm{FPh}-\mathrm{PhOMe}$ is favored by a factor of 80 based on the stability of the corresponding B structures, but by analyzing only barrier heights one would expect that the formation of $\mathrm{FPh}-\mathrm{PhF}$ is favored by a factor of 4 . Interestingly, the displacement of the methoxy group from the para- to the ortho- position destabilizes B to such a degree that the product distribution is inverted, which is also observed in the experiment. This destabilization can likely be attributed to repulsion between the oxygen atom in the methoxy group and the second aryl moiety. 


\section{Release of the coupling product}

The species $\mathbf{C}$ and $\mathbf{D}$ from Fig. 2 do not represent the final products. To model the release of the coupling product, we investigated a possible two-step mechanism that starts with a nucleophilic attack at the boron atom and proceeds with the dissociation of the coupling product from the tetrahedral complex. This is similar to nucleophilic substitutions at carbonyl groups. We studied this mechanism for the intermediate $\mathbf{D}$ obtained from $\mathrm{BPh}_{4}^{-}$using acetonitrile and water as nucleophiles. Our results obtained with B3LYP/6$31 \mathrm{G}(\mathrm{d})$ are summarized in Fig. 6, further results are available from Table 20 in the supporting information.

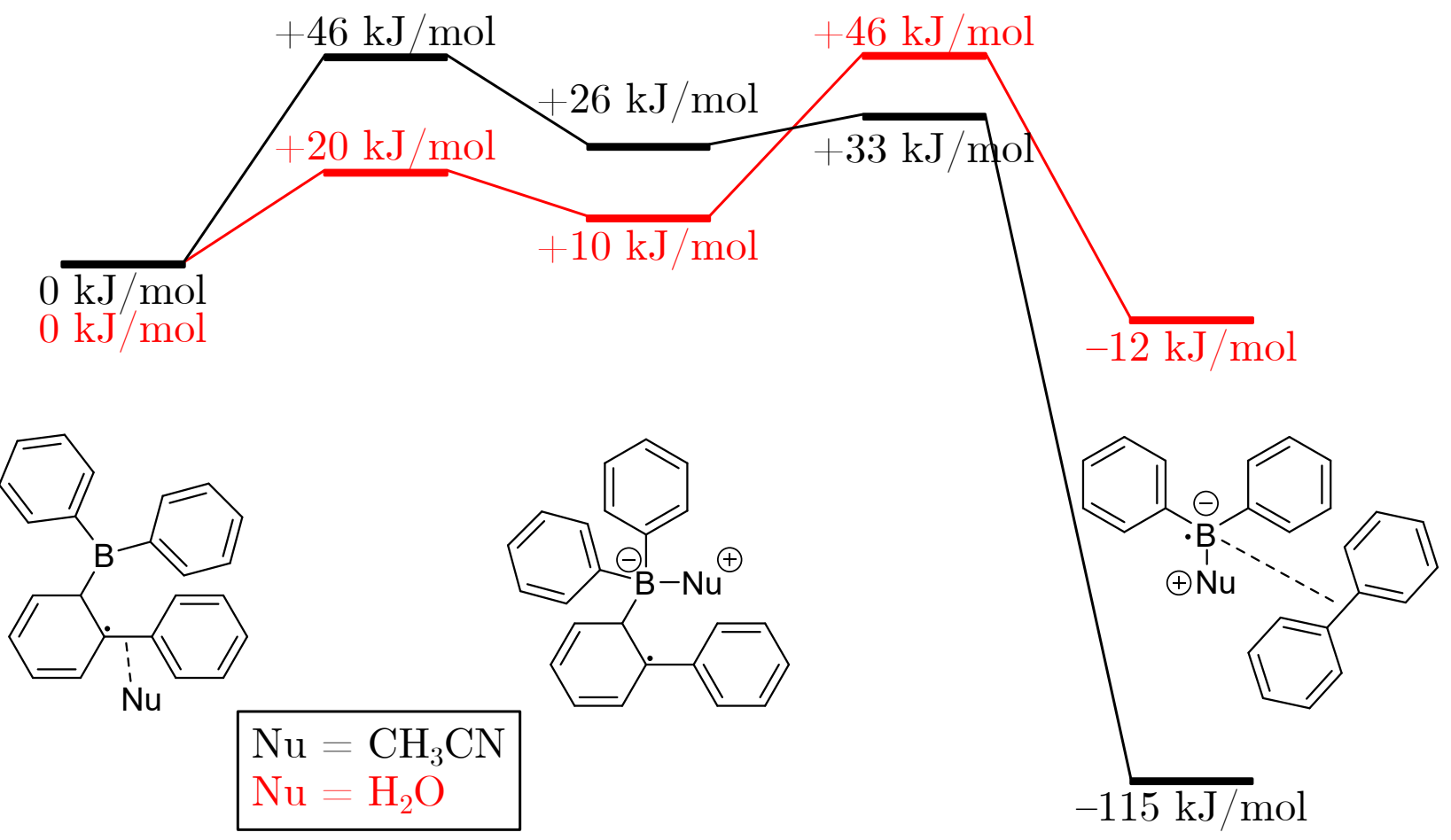

Figure 6: Schematic energy profile of the two-step substitution reaction that releases the final product. The reaction is studied with B3LYP/6-31G(d) for the intermediate $\mathbf{D}$ formed from tetraphenylborate. Acetonitrile and water are investigated as nucleophiles.

Distinctly different energy profiles are obtained with the two nucleophiles: While water forms the tetrahedral complex with a low barrier, this intermediate is higher in energy for acetonitrile $\left(20 \mathrm{~kJ} \mathrm{~mol}^{-1}\right.$ vs. $\left.46 \mathrm{~kJ} \mathrm{~mol}^{-1}\right)$. This may be due to the high affinity of boron to oxygen. The final product in which the diphenyl molecule is detached from the complex 
is, however, much lower in energy when acetonitrile is used as nucleophile $\left(12 \mathrm{~kJ} \mathrm{~mol}^{-1}\right.$ vs. $115 \mathrm{~kJ} \mathrm{~mol}^{-1}$ ). Since acetonitrile adopts a bent structure in the product, we attribute the lower final energy to the interaction of the $\mathrm{C} \equiv \mathrm{N} \pi^{*}$-orbitals with the radical electron. A similar stabilizing interaction cannot occur with water as nucleophile.

In previous experimental work, ${ }^{6,7}$ acetonitrile was used as solvent and water was added during the workup. It was observed that the presence of water in the reaction mixture facilitates the reaction. While we did not model an explicit substitution of one nucleophile by the other, our results in Fig. 6 suggest that if water and acetontrile are present at the same time, an initially attached water molecule will be replaced by acetonitrile eventually.

\section{Conclusion}

In this work, we have investigated the reaction mechanisms of aryl-aryl couplings and arylalkenyl couplings that follow the oxidation of quaternary borate salts. As suggested by our preceding studies, oxidation occurs preferably on the most electron-rich substituent. Whenever an alkenyl group is part of the coupling, the new C-C bond is formed without an energy barrier during the irreversible relaxation following oxidation. For these reactions, it is hardly possible to make a statement on the selectivity of the coupling because the new C-C bond is formed faster than any equilibration in the doublet state can occur. We observed, however, that couplings between two electron-deficient residues are often not possible when electron-rich residues are present, depending on the strength on the electron-withdrawing effects. Which residues are coupled in aryl-alkenyl couplings is likely determined by the point on the potential energy surface at which the oxidation occurs. To obtain further insight into this reaction, one would likely need to carry out ab initio molecular dynamics simulations.

In contrast, we found a three-membered ring as an intermediate in aryl-aryl couplings. The energy barriers to open such a ring are high compared to the barriers that separate the ring structures that can be formed between the different residues. As a consequence, 
the thermodynamically most favorable intermediate is formed before the reaction proceeds. Substituents on the aryl moieties influence the energy differences between the reaction intermediates and thus control the selectivity. The distribution of the coupling products is determined by an interplay between the stabilization of the pivotal three-membered ring and the rate with which it opens. If electron-rich substituents are coupled, the three-membered ring becomes more stable, the barrier towards ring opening increases, and the opening itself becomes more endergonic. For ortho-substituted residues, however, steric hindrance as well as possible bonding interactions between the boron atom and the substituent in ortho-position need to be considered as additional factors that can reverse trends expected from electronic effects.

After the ring opening, the boron residue stays coordinated and can migrate along the aryl residue, but only the migration to the ortho-position is associated with a significant energy gain. These secondary rearrangements are, however, hard to observe experimentally since the final reaction products remain the same independent of the position of the boron residue on the aryl ring. The subsequent release of the boron residue from the biphenyl system proceeds through a two-step nucleophilic substitution with an intermediate in which boron is tetrahedrally coordinated. The formation of the tetrahedral complex is associated with a low barrier when water is used as nucleophile, whereas the overall substitution reaction is more favorable with acetonitrile as nucleophile.

We tested different density functionals in this work and found that B3LYP delivers energy differences that are in acceptable agreement with EOM-IP-CCSD. The product distributions obtained from our B3LYP calculations reproduce the experimental trends qualitatively. As an alternative to B3LYP, the M06-2X functional with D3(0) correction and C-PCM solvation also works well. However, we also found that dispersion corrections and polarizable continuum models do not lead to a uniform improvement of the description of the reaction. 


\section{Acknowledgement}

The authors thank Prof. Hendrik Zipse and Prof. Jörg August Becker for helpful discussions. F.M. is grateful to the European Union for co-funding by the Erasmus + program. T.C.J. gratefully acknowledges funding from the European Research Council (ERC) under the European Union's Horizon 2020 research and innovation program (Grant Agreement No. 851766) and from the Fonds der Chemischen Industrie (FCI). D.D. and A.M. are grateful to the FCI, the Deutsche Forschungsgemeinschaft (DFG) (grant DI 2227/2-1), SFB 749, and the Ludwig-Maximilians University of Munich for PhD funding and financial support.

\section{Supporting Information Available}

The following files are available free of charge.

- Computational_insights_borate_salts_SI-energies.pdf: Further energy differences and activation barriers for a large variety of tetraorganoborates. Images of two B-asym type geometries.

- Computational_insights_borate_salts_SI-geometries.txt: Geometries of all molecules calculated during this work.

\section{References}

(1) Miyaura, N.; Suzuki, A. Palladium-Catalyzed Cross-Coupling Reactions of Organoboron Compounds. Chem. Rev. 1995, 95, 2457-2483.

(2) Miyaura, N.; Suzuki, A. Stereoselective synthesis of arylated (E)-alkenes by the reaction of alk-1-enylboranes with aryl halides in the presence of palladium catalyst. J. Chem. Soc., Chem. Commun. 1979, 866-867. 
(3) Harvey, J. N.; Daru, A. Computational Chemistry and Non-noble Metal-catalyzed Cross-coupling Reaction Mechanisms. Chimia 2020, 74, 467-469.

(4) Geske, D. H. The Electroöxidation of the Tetraphenylborate Ion; An Example of a Secondary Chemical Reaction Following the Primary Electrode Process. J. Phys. Chem. 1959, 63, 1062-1070.

(5) Beil, S. B.; Möhle, S.; Enders, P.; Waldvogel, S. R. Electrochemical instability of highly fluorinated tetraphenyl borates and syntheses of their respective biphenyls. Chem. Commun. 2018, 54, 6128-6131.

(6) Music, A.; Baumann, A. N.; Spieß, P.; Plantefol, A.; Jagau, T. C.; Didier, D. Electrochemical Synthesis of Biaryls via Oxidative Intramolecular Coupling of Tetra(hetero)arylborates. J. Am. Chem. Soc. 2020, 142, 4341-4348, PMID: 32040918.

(7) Baumann, A. N.; Music, A.; Dechent, J.; Müller, N.; Jagau, T. C.; Didier, D. ElectroOlefination-A Catalyst Free Stereoconvergent Strategy for the Functionalization of Alkenes. Chem.-Eur. J. 2020, 26, 8382-8387.

(8) Music, A.; Baumann, A.; Boser, F.; Müller, N.; Matz, F.; Jagau, T.-C.; Didier, D. Photocatalyzed Transition-Metal-Free Oxidative Cross-Coupling Reactions of Tetraorganoborates. Chem.-Eur. J. 2021, accepted manuscript.

(9) Gerleve, C.; Studer, A. Transition-Metal-Free Oxidative Cross-Coupling of Tetraarylborates to Biaryls Using Organic Oxidants. Angew. Chem. Int. Edit. 2020, 59, 1546815473.

(10) Frisch, M. J. et al. Gaussian 16 Revision C.01. 2016; Gaussian Inc. Wallingford CT.

(11) Shao, Y. et al. Advances in molecular quantum chemistry contained in the Q-Chem 4 program package. Mol. Phys. 2015, 113, 184-215. 
(12) Stephens, P. J.; Devlin, F. J.; Chabalowski, C. F.; Frisch, M. J. Ab Initio Calculation of Vibrational Absorption and Circular Dichroism Spectra Using Density Functional Force Fields. J. Phys. Chem. 1994, 98, 11623-11627.

(13) Behn, A.; Zimmerman, P. M.; Bell, A. T.; Head-Gordon, M. Efficient exploration of reaction paths via a freezing string method. J. Chem. Phys. 2011, 135, 224108.

(14) Chai, J.-D.; Head-Gordon, M. Systematic optimization of long-range corrected hybrid density functionals. J. Chem. Phys. 2008, 128, 084106.

(15) Staroverov, V. N.; Scuseria, G. E.; Tao, J.; Perdew, J. P. Comparative assessment of a new nonempirical density functional: Molecules and hydrogen-bonded complexes. $J$. Chem. Phys. 2003, 119, 12129-12137.

(16) Zhao, Y.; Truhlar, D. G. The M06 suite of density functionals for main group thermochemistry, thermochemical kinetics, noncovalent interactions, excited states, and transition elements: two new functionals and systematic testing of four M06-class functionals and 12 other functionals. Theor. Chem. Acc. 2008, 120, 215-241.

(17) Dasgupta, S.; Herbert, J. M. Standard grids for high-precision integration of modern density functionals: SG-2 and SG-3. J. Comput. Chem. 2017, 38, 869-882.

(18) Cossi, M.; Rega, N.; Scalmani, G.; Barone, V. Energies, structures, and electronic properties of molecules in solution with the C-PCM solvation model. J. Comput. Chem. 2003, 24, 669-681.

(19) Grimme, S.; Antony, J.; Ehrlich, S.; Krieg, H. A consistent and accurate ab initio parametrization of density functional dispersion correction (DFT-D) for the 94 elements H-Pu. J. Chem. Phys. 2010, 132, 154104.

(20) Grimme, S.; Ehrlich, S.; Goerigk, L. Effect of the damping function in dispersion corrected density functional theory. J. Comput. Chem. 2011, 32, 1456-1465. 
(21) Stanton, J. F.; Bartlett, R. J. The equation of motion coupled-cluster method. A systematic biorthogonal approach to molecular excitation energies, transition probabilities, and excited state properties. J. Chem. Phys. 1993, 98, 7029-7039.

(22) Shavitt, I.; Bartlett, R. J. Many-Body Methods in Chemistry and Physics: MBPT and Coupled-Cluster Theory; Cambridge University Press: Cambridge, UK, 2009.

(23) Evans, M. G.; Polanyi, M. Some applications of the transition state method to the calculation of reaction velocities, especially in solution. Trans. Faraday Soc. 1935, 31, 875-894. 